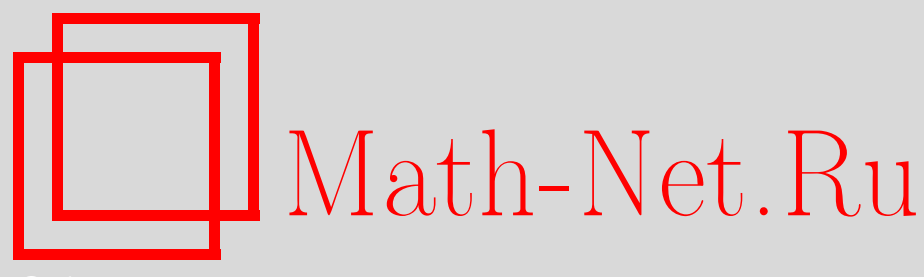

В. М. Калита, И. М. Иванова, В. М. Локтев, Квантовые эффекты при намагничивании легкоосного ферромагнетика с $S=1, T M \Phi, 2012$, том 173 , номер 2, 333-352

DOI: https://doi.org/10.4213/tmf6964

Использование Общероссийского математического портала Math-Net.Ru подразумевает, что вы прочитали и согласны с пользовательским соглашением http: //www . mathnet.ru/rus/agreement

Параметры загрузки:

IP : 18.208 .226 .222

26 апреля 2023 г., 13:11:28

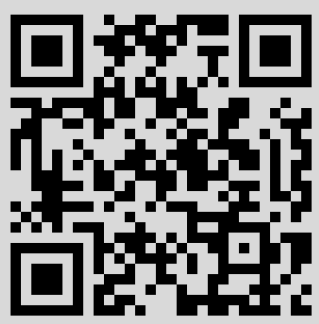




\title{
КВАНТОВЫЕ ЭФФЕКТЫ ПРИ НАМАГНИЧИВАНИИ ЛЕГКООСНОГО ФЕРРОМАГНЕТИКА С $S=1$
}

\begin{abstract}
Проанализировано влияние квантовых эффектов на магнитные свойства сильно анизотропного легкоосного ферромагнетика с одноионной анизотропией, сравнимой с обменным взаимодействием спинов с $S=1$. Показано, что в этом случае, несмотря на квантовый подход, применимо описание в духе теории фазовых переходов Ландау. Рассмотрены два случая намагничивания: продольным и поперечным внешними магнитными полями. Определены критические значения полей и найдены квантовые поправки, проявляющиеся в так называемом "сокращении" модуля среднего спина. Найдено, что при достаточно большой одноионной анизотропии угловая зависимость критического поля отклоняется от астроиды Стонера-Вольфарта.
\end{abstract}

Ключевые слова: квантовые фазовые переходы, одноионная анизотропия, намагничивание легкоосного ферромагнетика, критическое поле перемагничивания легкоосного ферромагнетика.

\section{1. ВВЕДЕНИЕ}

Несмотря на квантовую природу магнетизма, описание свойств магнитных систем - получение кривых намагничивания - проводится, как правило, в рамках квазиклассического подхода [1], [2]. В то же время известно, что роль квантовых эффектов важна, в частности, при описании магнитных свойств парамагнетиков (см., например, работы [3]-[7]). Такие эффекты также принципиальны и в случае формирования магнитного порядка в сильно анизотропных магнетиках с константой одноионной анизотропии (ОА), сравнимой по величине с константой обменного взаимодействия (ОВ) [8]-[19] (см. также книги [20], [21]). K ним, в частности, относятся ван-флековские магнетики с сильной ОА легкоплоскостного типа, в которых, как показано в работах [22]-[31], магнитное поле при $T=0$ индуцирует квантовые фазовые переходы (КФП) из синглетного состояния в магнитоупорядоченное.

${ }^{*}$ Институт физики НАН Украины, Киев, Украина. E-mail: vmkalita@ukr.net

${ }^{\dagger}$ Национальный технический университет КПИ, Киев, Украина. E-mail: maivanov@ukr.net

${ }^{\ddagger}$ Институт теоретической физики им. Н. Н. Боголюбова НАН Украины, Киев, Украина. E-mail: vloktev@bitp.kiev.ua 
В одноосном ферромагнетике $(\Phi \mathrm{M})$ со слабой ОА процесс намагничивания согласно общепринятым представлениям может быть описан в рамках квазиклассического приближения, когда под действием магнитного поля вектор среднего спина на узле изменяет лишь свое направление при сохранении модуля. Соответственно изучение таких фазовых переходов ведется в рамках теории Ландау, параметрами порядка в которой служат направляющие углы векторов средней намагниченности [2],[32], [33].

В пределе бесконечно большой легкоосной ОА имеет место уже другой предельный случай, отвечающий модели Изинга, когда квазиклассический подход также применим, но с противоположным условием: спины в каждом узле, как и намагниченность в целом, сохраняют свой модуль, однако ориентированы исключительно вдоль легкой оси [34].

В случае же ФМ с конечной ОА учет квантовых эффектов является, как будет видно, необходимым. Ниже рассматривается перемагничивание легкоосного ФМ с большой, но конечной ОА. Будет показано, что в таком ФМ квантовые эффекты, которые проявляются как "сокращение" модуля вектора среднего спина, имеют место не только в поперечном (перпендикулярном легкой оси), но и в продольном (параллельном легкой оси) магнитных полях. В литературе (см., например, обзор [14] или книгу [21]) описано сокращение величины среднего спина в основном состоянии ФМ с легкоплоскостным либо двухосным типом ОА. Такое сокращение есть не что иное, как следствие конкуренции ОВ и ОА. В то же время в ФМ с легкоосной ОА сокращения спина в основном состоянии не происходит, и среднее значение проекции спина равно его предельной величине, хотя известно, что ОА легкоосного типа изменяет спектр спиновых возбуждений [4]. Во внешнем магнитном поле, направленном перпендикулярно легкой оси, из-за некоммутации зеемановского и анизотропного взаимодействий при отклонении среднего спина от легкой оси сначала происходит уменьшение среднего спина, а затем, при дальнейшем возрастании поля, среднее значение спина возрастает вплоть до максимального [8]. Таким образом, при отклонении среднего спина от легкой оси ФМ с сильной ОА проявляет себя эффект квантового сокращения спина.

Поэтому очевидно, что подобный эффект должен иметь место и в случае ориентационного фазового перехода, происходящего в легкоосном ФМ с ОА в продольном поле. Действительно, при ориентационном фазовом переходе в критическом поле происходит опрокидывание магнитного момента ФМ путем однородного разворота (когерентного вращения) спинов. В ходе этого перехода спины отклоняются от легкой оси, и квантовое сокращение намагниченности не может не появиться. Однако наиболее распространенный в литературе подход к описанию ФМ с ОА основан на нахождении спиновых конфигураций с использованием процедуры самосогласования [8]-[15], [17]-[21]. В этом приближении не удается аккуратно установить, как изменяется модуль среднего спина при его опрокидывании в ходе ориентационного фазового перехода, так как такое приближение справедливо только для равновесных состояний [35]. Подчеркнем также, что границы устойчивости фаз магнетиков с сильной OА часто находят с помощью магнонных спектров, получаемых, например, с использованием техники операторов Хаббарда [8], [11]-[15], [17]-[21]. 
Следует иметь в виду, что в случае ФМ с легкоосной ОА критическое поле соответствующего перехода равно полю потери устойчивости ФМ-фазы. Однако, если OA превосходит ОВ, то в процессе перемагничивания однородный разворот с когерентным вращением спинов уже невозможен, и (в отличие от рассмотренного в работах [2], [32], [33]) индуцируемый полем фазовый переход оказывается магнитным КФП І рода. Если же магнитное поле поперечно, то в ФМ с ОА становится возможным лишь спин-ориентационный фазовый переход II рода, обусловленный равновесным разворотом вектора намагниченности с несохранением его модуля.

Поскольку квантовые эффекты наиболее существенны при низких температу$\operatorname{pax}[36]$, описание намагничивания одноосного ФМ проведем для случая $T=0$, предполагая "затравочный" спин на узле $S=1$. При этом процесс намагничивания будет рассматриваться с использованием формализма Ландау, когда равновесное состояние находится путем минимизации энергии основного состояния, однако не по параметру порядка, а по коэффициентам разложения волновой функции по спиновым функциям [29]-[31]. Отметим, что нахождение функции основного состояния и варьирование по ее параметрам использовалось также в работах [37], [38], где, однако, эффект квантового сокращения спина ввиду изотропности модели отсутствовал, а сама задача фактически свелась к обычному описанию с направляющими классическими векторами для параметра порядка спинового нематика. Хотя квантовые эффекты в системах с сильной ОА неоднократно анализировались при рассмотрении динамики спиновых возбуждений (см., например, работы [8], [9], [11]-[15], [17]-[21]), для одноосного ФМ с сильной ОА описания фазовых переходов в рамках теории Ландау, насколько известно, не проводилось. Подчеркнем также, что с помощью этого подхода удалось понять, как ОА модифицирует кривую границ устойчивости ФМ-фазы в наклонных магнитных полях, известную как астроида Стонера-Вольфарта [39], [40].

\section{2. ГАМИЛЬТОНИАН}

Пусть имеется система спинов с $S=1$, ОА легкоосного типа и изотропным ОВ. Гамильтониан такой системы имеет стандартный и достаточно простой вид:

$$
\widehat{H}=-D \sum_{\mathbf{n}}\left(S_{\mathbf{n}}^{Z}\right)^{2}-J \sum_{\mathbf{n}, \mathbf{m}} \mathbf{S}_{\mathbf{n}} \mathbf{S}_{\mathbf{m}}-\mathbf{H} \sum_{\mathbf{n}} \mathbf{S}_{\mathbf{n}}
$$

где $D>0$ - константа OA, $J>0$ - константа OB, $\mathbf{S}_{\mathbf{n}}, \mathbf{S}_{\mathbf{m}}$ - операторы спинов с позициями, задаваемыми векторами $\mathbf{n}$ и $\mathbf{m}$, причем предполагается, что ОВ отлично от нуля только для ближайших соседей, $Z$ - легкая ось, а $\mathbf{H}$ - внешнее магнитное поле.

Рассчитаем энергию основного состояния системы (1). Будем при этом использовать простейшее приближение квантового самосогласованного поля, полагая, что в основном состоянии для средних от произведений справедливо равенство

$$
\left\langle\mathbf{S}_{\mathbf{n}} \mathbf{S}_{\mathbf{m}}\right\rangle=\left\langle\mathbf{S}_{\mathbf{n}}\right\rangle\left\langle\mathbf{S}_{\mathbf{m}}\right\rangle .
$$

Учитывая, что в однородном ФМ узлы эквивалентны, или $\left\langle\mathbf{S}_{\mathbf{n}}\right\rangle=\langle\mathbf{S}\rangle$ и $\left\langle\left(S_{\mathbf{n}}^{Z}\right)^{2}\right\rangle=$ $\left\langle S_{Z}^{2}\right\rangle$, запишем выражение для энергии его основного состояния:

$$
E_{\mathrm{gr}}=-D\left\langle S_{Z}^{2}\right\rangle-I\langle\mathbf{S}\rangle^{2}-\mathbf{H}\langle\mathbf{S}\rangle,
$$


где $I=J z$ - константа, в которой $z$ - число ближайших соседей. В выражении (2) мы пренебрегли межузельными флуктуациями, однако действие ОА ниже учитывается точно. Подобный подход оправдан в случае достаточно сильной анизотропии, когда перемагничивание требует больших полей.

В принятом приближении волновая функция основного состояния ФМ мультиплицируется и имеет вид прямого произведения одноузельных волновых функций. Последние эквивалентны и для $S=1$ и могут быть представлены в общем виде:

$$
\psi=e^{i \varphi+} C_{+}|+1\rangle+C_{0}|0\rangle+e^{-i \varphi_{-}} C_{-}|-1\rangle,
$$

где коэффициенты $C_{j}$ вещественны и удовлетворяют нормировке $\sum_{j} C_{j}^{2}=1$.

Функция (3) действительно является общей, поскольку известно, что спин $S=1$ описывается унитарной группой $S U(3)$, содержащей 8 независимых параметров, а именно проекции $S_{j}$ спина и компоненты квадрупольного спинового момента $Q_{j k}=$ $\left(S_{j} S_{k}+S_{k} S_{j}\right) / 2$. Однако с учетом нормировки волновая функция (3) оставляет только 4 (а не 6, как утверждается в работе [41]) независимых переменных, которых достаточно для полного описания поведения такого ФМ. С помощью функции (3) легко найти все имеющиеся средние:

$$
\begin{aligned}
\left\langle S_{Z}\right\rangle & =C_{+}^{2}-C_{-}^{2}, \quad\left\langle S_{x}\right\rangle=\sqrt{2\left(1-C_{+}^{2}-C_{-}^{2}\right)}\left(C_{+} \cos \varphi_{+}+C_{-} \cos \varphi_{-}\right), \\
\left\langle S_{y}\right\rangle & =\sqrt{2\left(1-C_{+}^{2}-C_{-}^{2}\right)}\left(C_{+} \sin \varphi_{+}+C_{-} \sin \varphi_{-}\right), \\
\left\langle Q_{z z}\right\rangle & =C_{+}^{2}+C_{-}^{2}, \quad\left\langle Q_{x x}\right\rangle=\frac{1}{2}\left(2-C_{+}^{2}-C_{-}^{2}+2 C_{+} C_{-} \cos \left(\varphi_{+}+\varphi_{-}\right)\right), \\
\left\langle Q_{y y}\right\rangle & =\frac{1}{2}\left(2-C_{+}^{2}-C_{-}^{2}-2 C_{+} C_{-} \cos \left(\varphi_{+}+\varphi_{-}\right)\right), \\
\left\langle Q_{x y}\right\rangle & =2 C_{+} C_{-} \sin \left(\varphi_{+}+\varphi_{-}\right), \\
\left\langle Q_{x z}\right\rangle & =\frac{1}{\sqrt{2}} \sqrt{1-C_{+}^{2}-C_{-}^{2}}\left(C_{+} \cos \varphi_{+}-C_{-} \cos \varphi_{-}\right), \\
\left\langle Q_{y z}\right\rangle & =\frac{1}{\sqrt{2}} \sqrt{1-C_{+}^{2}-C_{-}^{2}}\left(C_{+} \sin \varphi_{+}-C_{-} \sin \varphi_{-}\right) .
\end{aligned}
$$

Видно, что средние значения диагональных компонент квадрупольного спинового момента (4), как и положено, удовлетворяют равенству $\sum_{J}\left\langle Q_{j j}\right\rangle=2$. В результате искомые средние выражаются через 4 параметра, которыми могут быть выбраны коэффициенты $C_{+}$и $C_{-}$, а также их фазы $\varphi_{+}$и $\varphi_{-}$волновой функции $(3)$.

Ее использование приводит выражение (2) к виду

$$
\begin{aligned}
E_{\mathrm{gr}}= & -D\left(C_{+}^{2}+C_{-}^{2}\right)-I\left[\left(C_{+}^{2}-C_{-}^{2}\right)^{2}+\right. \\
& \left.+2\left(1-C_{+}^{2}-C_{-}^{2}\right)\left(C_{+}^{2}+C_{-}^{2}+2 C_{+} C_{-} \cos \left(\varphi_{+}-\varphi_{-}\right)\right)\right]- \\
& -\sqrt{2} H_{x} \sqrt{1-C_{+}^{2}-C_{-}^{2}}\left(C_{+} \cos \varphi_{+}+C_{-} \cos \varphi_{-}\right)- \\
& -\sqrt{2} H_{y} \sqrt{1-C_{+}^{2}-C_{-}^{2}}\left(C_{+} \sin \varphi_{+}+C_{-} \sin \varphi_{-}\right)-H_{z}\left(C_{+}^{2}-C_{-}^{2}\right),
\end{aligned}
$$

где $\mathbf{H}=\left(H_{x}, H_{y}, H_{z}\right)$. 
Как видно, вклад в выражение (5) от ОВ имеет наименьшую величину при $\cos \left(\varphi_{+}-\varphi_{-}\right)=1$. Если $\varphi_{+}=\varphi_{-}$, угол $\varphi_{+}$(либо $\left.\varphi_{-}\right)$приобретает геометрический смысл направляющего угла для проекций $\left\langle S_{x}\right\rangle,\left\langle S_{y}\right\rangle$. В этом случае величины $\varphi_{ \pm}$, минимизирующие вклад энергии Зеемана в выражение (5), определяются из условия $\operatorname{tg} \varphi_{ \pm}=H_{y} / H_{x}$, и для поперечных легкой оси проекций выполняется соотношение $\left\langle S_{x}\right\rangle /\left\langle S_{y}\right\rangle=H_{x} / H_{y}$.

В то же время лабораторную систему координат удобно выбрать так, чтобы ось $Y$ была перпендикулярна плоскости, в которой лежат легкая ось $Z$ и вектор Н. В этом случае $H_{y}=0$ и соответственно $\varphi_{ \pm}=0$. В такой системе координат энергия основного состояния приобретает вид

$$
\begin{aligned}
E_{\mathrm{gr}}= & -D\left(C_{+}^{2}+C_{-}^{2}\right)-I\left[\left(C_{+}^{2}-C_{-}^{2}\right)^{2}+2\left(1-C_{+}^{2}-C_{-}^{2}\right)\left(C_{+}+C_{-}\right)^{2}\right]- \\
& -\sqrt{2} H_{x}\left(C_{+}+C_{-}\right) \sqrt{1-C_{+}^{2}-C_{-}^{2}}-H_{z}\left(C_{+}^{2}-C_{-}^{2}\right) .
\end{aligned}
$$

Минимизируя выражение (6) по коэффициентам $C_{+}$и $C_{-}$подобно тому, как это делалось в работе [30], найдем уравнения для определения основного состояния системы, которое, как известно, является стационарным:

$$
\begin{aligned}
\frac{\partial E_{\mathrm{gr}}}{\partial C_{+}}= & -2 D C_{+}-2 I\left[C_{+}\left(C_{+}^{2}-C_{-}^{2}\right)+\right. \\
& \left.+2\left(1-C_{+}^{2}-C_{-}^{2}\right)\left(C_{+}+C_{-}\right)-2 C_{+}\left(C_{+}+C_{-}\right)^{2}\right]- \\
& -\sqrt{2} H_{x}\left(\sqrt{1-C_{+}^{2}-C_{-}^{2}}-\frac{C_{+}\left(C_{+}+C_{-}\right)}{\sqrt{1-C_{+}^{2}-C_{-}^{2}}}\right)-2 H_{z} C_{+}=0, \\
\frac{\partial E_{\mathrm{gr}}}{\partial C_{-}}= & -2 D C_{-}-2 I\left[C_{-}\left(C_{-}^{2}-C_{+}^{2}\right)+\right. \\
& \left.+2\left(1-C_{+}^{2}-C_{-}^{2}\right)\left(C_{+}+C_{-}\right)-2 C_{-}\left(C_{+}+C_{-}\right)^{2}\right]- \\
& -\sqrt{2} H_{x}\left(\sqrt{1-C_{+}^{2}-C_{-}^{2}}-\frac{C_{-}\left(C_{+}+C_{-}\right)}{\sqrt{1-C_{+}^{2}-C_{-}^{2}}}\right)+2 H_{z} C_{-}=0 .
\end{aligned}
$$

При определении минимума $E_{\mathrm{gr}}$ нужно учитывать, что коэффициенты $C_{ \pm}$ограничены условием $0 \leqslant C_{ \pm} \leqslant 1$.

Как видно из формул (6)-(8), с помощью этого подхода при определении спиновых конфигураций не нужно решать математически более сложную и более громоздкую задачу самосогласования, как это делается в работах [8]-[15], [17]-[21], а определение границы устойчивости фазы не требует рассчета и магнонного спектра.

Таким образом, как следует из формул (6)-(8), задача поиска спиновых конфигураций в квантовом подходе сводится к минимизации энергии основного состояния $E_{\text {gr }}$ и тем самым действительно удовлетворяет теории Ландау фазовых переходов, хотя в квантовом случае вариационными, как отмечалось, являются коэффициенты $C_{j}$ и их фазы (см. формулу $\left.(3)\right)$.

\section{3. СОБСТВЕННАЯ СИСТЕМА КООРДИНАТ}

При описании одноосной системы (1) можно воспользоваться ее симметрийными свойствами. Для этого введем собственную систему координат с осями $\xi, \eta, \zeta$ так, 
чтобы средний спин был направлен вдоль оси $\zeta$, а ось $\xi$ лежала в плоскости, образованной осью $Z$ и вектором Н. В собственной системе координат составляющие среднего спина, поперечные к оси $\zeta$, согласно условиям $\left\langle S_{\xi}\right\rangle=\left\langle S_{\eta}\right\rangle=0$ отсутствуют и соответственно симметрии магнитного состояния системы должны выполняться равенства $\left\langle Q_{\xi \eta}\right\rangle=\left\langle Q_{\zeta \eta}\right\rangle=0$. С помощью выражений (4) легко убедиться, что при оговоренных ограничениях вид волновой функции спина в собственной системе координат упрощается до (см. работы [8], [12], [14])

$$
\psi=\cos \phi|+1\rangle+\sin \phi|-1\rangle \text {. }
$$

Нужно при этом иметь в виду, что функция (9) отвечает оси квантования, направленной вдоль среднего спина.

В собственной системе средние (4) также изменяются:

$$
\left\langle S_{\zeta}\right\rangle=\cos (2 \phi), \quad\left\langle Q_{\zeta \zeta}\right\rangle=1, \quad\left\langle Q_{\xi \xi}\right\rangle=\frac{1}{2}(1+\sin (2 \phi)), \quad\left\langle Q_{\eta \eta}\right\rangle=\frac{1}{2}(1-\sin (2 \phi)) .
$$

Переход в такую систему координат без потери общности задается поворотом вокруг оси $Y \| \eta$ на геометрический угол $\theta$. Тогда энергия (2) как функция средних приводится к виду

$$
E_{\mathrm{gr}}=-I\left\langle S_{\zeta}\right\rangle^{2}-D\left(\left\langle S_{\zeta}^{2}\right\rangle \cos ^{2} \theta+\left\langle S_{\xi}^{2}\right\rangle \sin ^{2} \theta\right)-H_{\|}\left\langle S_{\zeta}\right\rangle \cos \theta-H_{\perp}\left\langle S_{\zeta}\right\rangle \sin \theta,
$$

где $H_{\|} \equiv H_{z}$ - параллельная, а $H_{\perp}$ - перпендикулярная составляющие магнитного поля относительно исходной легкой оси ФМ. Обратим внимание, что переход в собственную систему координат в ФМ оставляет инвариантным изотропное ОВ, т. е. изменения касаются лишь слагаемых энергии, связанных с ОА и с полем.

С использованием равенств (10) выражение (11) можно записать как функцию лишь углов:

$$
\begin{aligned}
E_{\mathrm{gr}}(\theta, \phi)= & -I \cos ^{2}(2 \phi)-D\left(\cos ^{2} \theta+\frac{\sin ^{2} \theta}{2}(1+\sin (2 \phi))\right)- \\
& -H_{\|} \cos (2 \phi) \cos \theta-H_{\perp} \cos (2 \phi) \sin \theta
\end{aligned}
$$

В результате спиновые конфигурации основного состояния и их взаимное превращение в магнитном поле определяются из уравнений (ср. формулы (6) и (7))

$$
\begin{array}{rl}
\frac{\partial E_{\mathrm{gr}}(\theta, \phi)}{\partial \phi}=4 & I \cos (2 \phi) \sin (2 \phi)-D \sin ^{2} \theta \cos (2 \phi)+ \\
& +2 H_{\|} \sin (2 \phi) \cos \theta+2 H_{\perp} \sin (2 \phi) \sin \theta=0, \\
\frac{\partial E_{\mathrm{qr}}(\theta, \phi)}{\partial \theta}=D & \sin \theta \cos \theta(1-\sin (2 \phi))+ \\
& +H_{\|} \cos (2 \phi) \sin \theta-H_{\perp} \cos (2 \phi) \cos \theta=0,
\end{array}
$$

обобщающих квазиклассические уравнения тем, что наряду с геометрическим углом $\theta$ последовательно включают угол "смешивания" $\phi$.

Заметим, что выражение (5) для $E_{\text {gr }}$ было получено в самом общем виде с использованием волновой функции (3), тогда как при записи $E_{\mathrm{gr}}$ в форме (12) были 


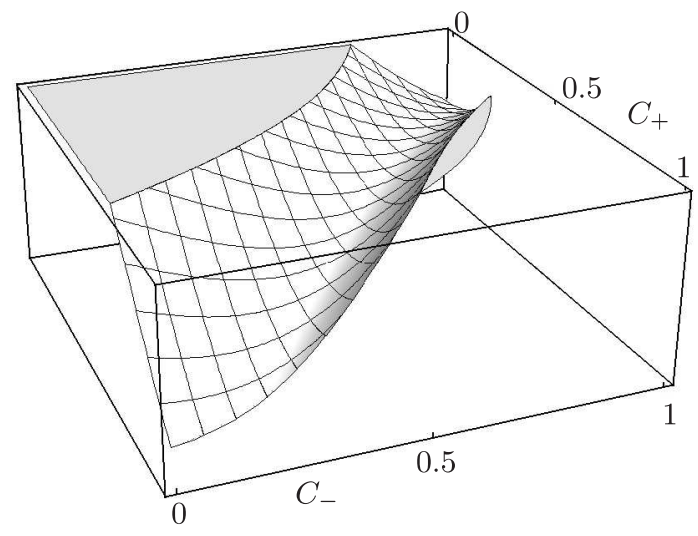

Рис. 1. Энергетический профиль $E_{\mathrm{gr}}\left(C_{+}, C_{-}\right)$при $D / I=1$ и $H_{\|} / I=0.25$.

использованы симметрийные свойства конкретного магнитного состояния рассматриваемой одноосной системы. Таким образом, подход, основанный на использовании волновой функции (3), является общим и применимым для любых систем с $S=1$. Кроме этого, подчеркнем, что записанная в виде $(6)$ энергия $E_{\mathrm{gr}}\left(C_{+}, C_{-}\right)$ и записанная в виде $(12)$ энергия $E_{\mathrm{gr}}(\theta, \phi)$ в одинаковой мере пригодны к описанию намагничивания системы (1). Поэтому при изучении намагничивания в продольном и поперечном полях мы будем обращаться к обоим выражениям для $E_{\mathrm{gr}}$. Однако для намагничивания в продольном поле чаще будет использоваться выражение (6) оно для этого случая более наглядно, а с выражением (12) удобнее работать в случае поперечного поля, что к тому же оказывается и проще.

\section{4. ПРОДОЛЬНОЕ ПОЛЕ}

Энергия $E_{\mathrm{gr}}\left(C_{+}, C_{-}\right)$в поле $\mathbf{H} \uparrow \uparrow Z\left(H_{z}=H_{\|} \neq 0, H_{x} \equiv H_{\perp}=0\right)$ может иметь два минимума, которые отвечают двум ФМ-состояниям - устойчивому и неустойчивому. В первом $C_{+}=1, C_{-}=0,\left\langle S_{z}\right\rangle \uparrow \uparrow H_{\|}$и $\left\langle S_{z}\right\rangle=1$, а его энергия $E_{\mathrm{gr}}^{\text {(st) }}=-D-I-H_{\|}$. В неустойчивом ФМ-состоянии $C_{+}=0, C_{-}=1,\left\langle S_{z}\right\rangle \uparrow \downarrow H_{\|}$и $\left\langle S_{z}\right\rangle=-1$, и его энергия $E_{\mathrm{gr}}^{\text {(unst) }}=-D-I+H_{\|}$. Устойчивому ФМ-состоянию отвечает минимум в точке $\theta=0$ и $\phi=0$ для энергии $E_{\mathrm{gr}}(12)$. Для этого состояния ось квантования направлена вдоль поля и $\left\langle S_{\zeta}\right\rangle=\cos (2 \phi)=1$. В неустойчивом ФМ-состоянии $\theta=\pi$ и $\phi=0$ и, следовательно, ось квантования противоположна магнитному полю $\zeta \uparrow \downarrow$ $H_{\|},\left\langle S_{\zeta}\right\rangle=\cos (2 \phi)$.

На рис. 1 изображен энергетический профиль $E_{\mathrm{gr}}\left(C_{+}, C_{-}\right)$, полученный численно для $D / I=1$ и $H_{z} / I=0.25$. Два минимума с $C_{+}=1, C_{-}=0$ и с $C_{+}=0$, $C_{-}=1$ (им отвечают противоположные направления намагниченности) разделены барьером, которому на профиле энергии $E_{\mathrm{gr}}\left(C_{+}, C_{-}\right)$отвечает седловая точка $C_{+} \neq 0, C_{-} \neq 0$. При $H_{\|} \rightarrow 0$ положение седловой точки симметрично относительно минимумов и имеет координаты $C_{ \pm}=1 / 2$. 


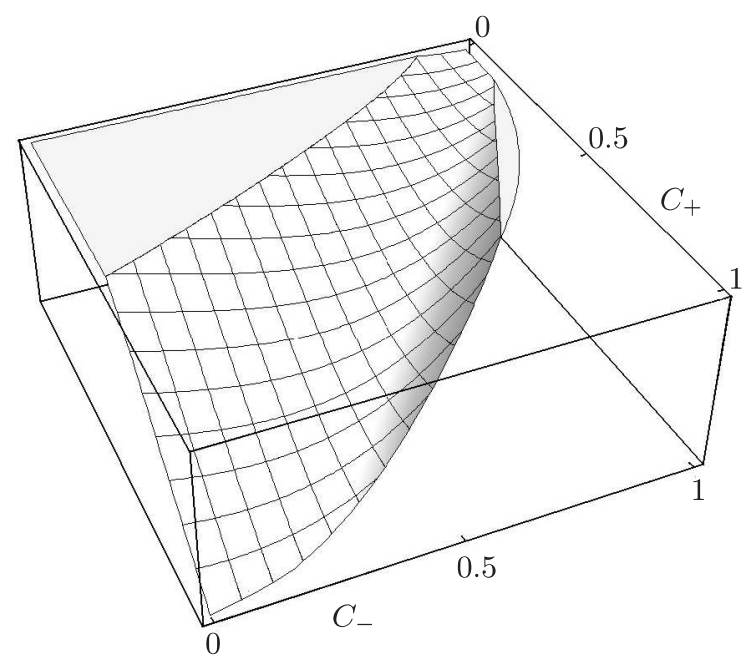

Рис. 2. Энергетический профиль $E_{\mathrm{gr}}\left(C_{+}, C_{-}\right)$при $D / I=1$ и $H_{\|}=H_{\mathrm{cr}}^{\|}=D$.

Из профиля $E_{\mathrm{gr}}$ можно найти критическое поле $H_{\mathrm{cr}}^{\|}$, в котором исчезает неустойчивое ФМ-состояние. Иными словами, в этом поле исчезает минимум на зависимости $E_{\mathrm{gr}}$ с намагниченностью, направленной против магнитного поля. Величина $H_{\mathrm{cr}}^{\|}$соответствует коэрцитивной силе при перемагничивании однодоменного ФМ в продольном поле, а сам процесс перемагничивания характеризуется гистерезисом с прямоугольной формой петли.

На рис. 2 показан энергетический профиль $E_{\mathrm{gr}}\left(C_{+}, C_{-}\right)$для значения $D / I=1$, когда внешнее магнитное поле $H_{\|}=H_{\mathrm{cr}}^{\|}$. Видно, что на поверхности $E_{\mathrm{gr}}\left(C_{+}, C_{-}\right)$ седловая точка уже отсутствует, а энергия при переходе от неустойчивого ФМ-состояния к устойчивому изменяется непрерывно и имеет вид "желоба". Зависимость $H_{\mathrm{cr}}^{\|}$ от величины ОА нетрудно получить численно. Соответствующий график зависимости $H_{\mathrm{cr}}^{\|}(D)$ приведен на рис. 3 и имеет два прямолинейных участка. На первом из них при $D / 2 I<1$ величина $H_{\text {сr }}^{\|}$зависит только от ОА. На втором, когда $D / 2 I>1$, величина $H_{\mathrm{cr}}^{\|}$уже не зависит от величины $D$, а определяется только ОВ.

Обсудим поведение системы в критическом поле, когда константа ОА удовлетворяет неравенству $D / 2 I<1$. Если предположить, что переходу системы из состояния с неустойчивой ориентацией намагниченности в состояние с направлением намагниченности вдоль поля отвечает кривая в пространстве $C_{+}, C_{-}$с наименьшими значениями энергии на энергетическом профиле (другими словами, система перемещается по градиенту поверхности $E_{\mathrm{gr}}\left(C_{+}, C_{-}\right)$или, как говорилось выше, вдоль дна "желоба" этого профиля), то при $D / 2 I \ll 1$ траекторией перехода является прямая

$$
C_{-}=1-C_{+}
$$

На ней вектор среднего спина, модуль которого, будучи равным своему квазиклассическому значению - единице, лишь изменяет свое направление:

$$
|\mathbf{S}|^{2}=\left\langle S_{x}\right\rangle^{2}+\left\langle S_{z}\right\rangle^{2}=1
$$




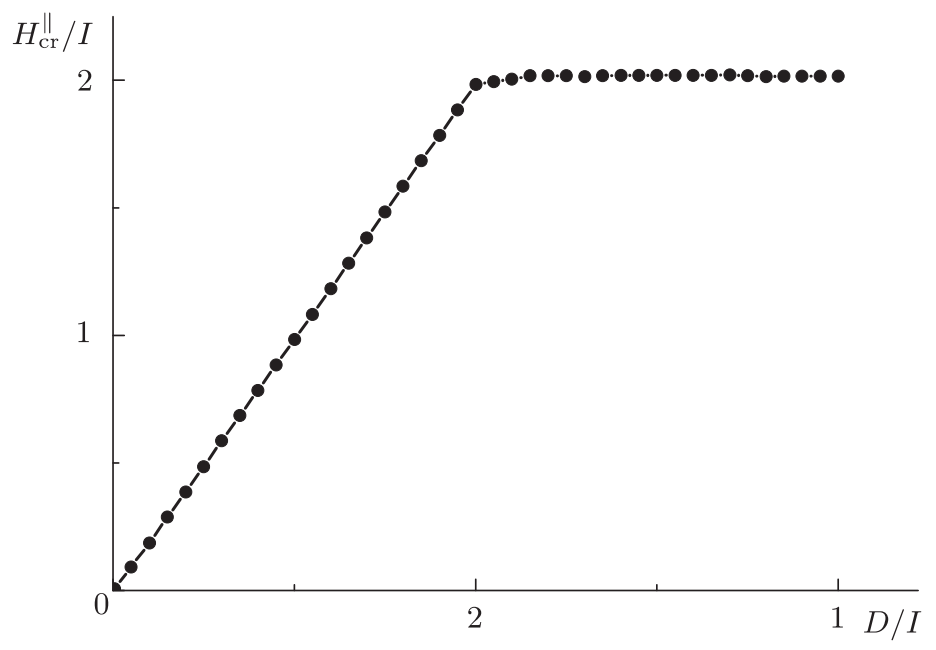

Рис. 3. Зависимость критического поля от одноионной анизотропии $H_{\mathrm{cr}}^{\|}(D)$.

С помощью формулы (16) легко убедиться, что седловая точка, разделяющая минимумы, имеет координаты

$$
C_{ \pm}^{(s)}=\frac{1}{2}\left(1 \mp \frac{H_{\|}}{D}\right) .
$$

Кроме этого, учтем, что на прямой (15) для проекции спина выполняется соотношение $\left\langle S_{z}\right\rangle=2 C_{+}-1$. Тогда легко записать выражение для энергии, относящейся к дну "желоба", в виде функции от $\left\langle S_{z}\right\rangle$ :

$$
E_{\mathrm{gr}}=-I-\frac{1}{2} D\left(1+\left\langle S_{z}\right\rangle^{2}\right)-H_{\|}\left\langle S_{z}\right\rangle .
$$

Поскольку модуль среднего спина для этих значений $C_{+}$и $C_{-}$также определяется формулой (16), то выражение (18) приобретает вид

$$
E_{\mathrm{gr}}=-I-\frac{1}{2} D\left(1+\cos ^{2} \theta\right)-H_{\|} \cos \theta
$$

где $\theta$ - полярный угол между осью $\zeta$ собственной системы и осью $Z$ лабораторной.

В итоге приходим к очевидному результату: в случае слабой $\mathrm{OA}, D / 2 I \ll 1$, при переходе из неустойчивого к устойчивому ФМ-состоянию выражение для энергии $E_{\mathrm{gr}}$ в критическом поле имеет такой же вид, как и при феноменологическом описании, соответствующем, в частности, теории Акулова [2]. Заметим, однако, что квазиклассическое приближение $\left\langle S_{z}^{2}\right\rangle=\left\langle S_{z}\right\rangle^{2}$ в выражении (1) дает при $\cos ^{2} \theta$ константу анизотропии вдвое большую, чем в формуле (19).

Выражение (19) можно также получить, если принять, что в случае $D / 2 I \ll 1$ квантовое "сокращение" среднего модуля спина не происходит и выполняется равенство $\left\langle S_{\zeta}\right\rangle=\cos (2 \phi)=1$. При подстановке последнего в энергию $E_{\mathrm{gr}}(\theta, \phi)$, записанную в виде (12), также придем к выражению (19). Другими словами, при 


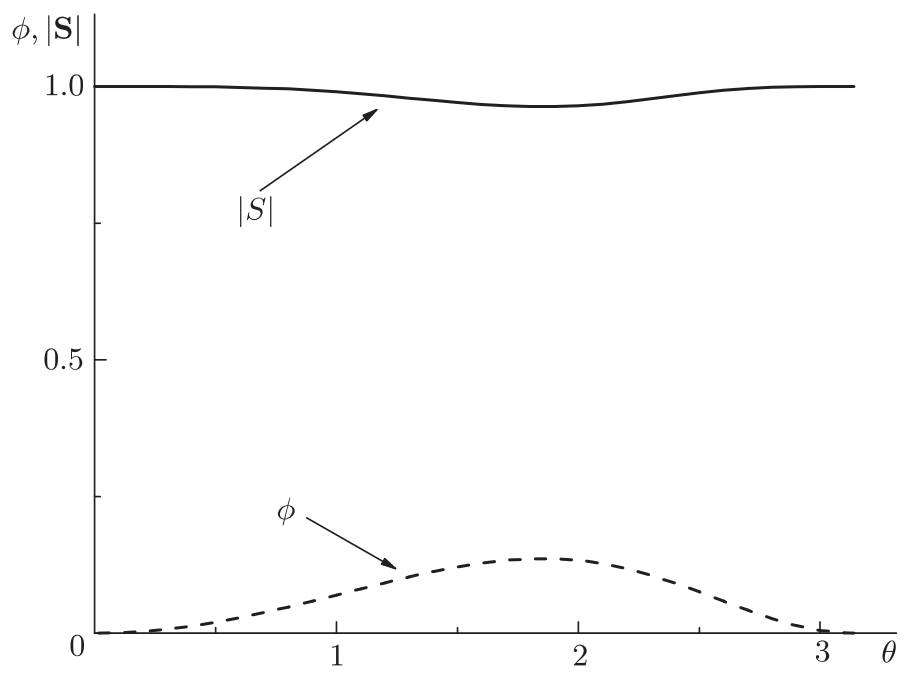

Рис. 4. Графики зависимостей $|\mathbf{S}(\theta)|$ и $\phi(\theta)$, полученные при тех же условиях, что и энергия на рис. 2.

квазиклассическом $(D / 2 I \ll 1)$ развороте среднего спина, когда параметры волновой функции связаны соотношением (15), спиновые проекции прямо задаются, как и должно быть, функциями $\cos \theta$ и $\sin \theta$.

В случае относительно больших $D / 2 I$ ситуация усложняется. Кривая, идущая по дну "желоба" от неустойчивого к устойчивому ФМ-состоянию, уже не будет лежать на прямой (15), что видно из рис. 2. Теперь при развороте среднего спина в критическом поле происходит сокращение его модуля (угловая зависимость последнего показана на рис. 4 - она получена при тех же условиях, что и энергия на рис. 2). На том же рис. 4 приведена зависимость $\phi(\theta)$, из которой видно, что сокращение среднего спина происходит несимметрично: оно больше в начале процесса отклонения намагниченности от исходного направления и несколько уменьшается в конце (напомним, что исходным является неустойчивое состояние с $\theta=\pi$ ). При $\theta \rightarrow \pi / 2$ упомянутое сокращение составляет всего $5 \%$.

При предельно большой ОА (в частности, при $D / 2 I>1$ ) поведение системы в критической точке $H_{\|}=H_{\text {cr }}^{\|}$кардинально изменяется. В самом деле, запишем одночастичный гамильтониан среднего поля [35]:

$$
H_{0}=-D S_{z}^{2}-2 I\left\langle S_{z}\right\rangle S_{z}-H_{z} S_{z}
$$

При $\left\langle S_{z}\right\rangle=1$, когда средний спин направлен против магнитного поля, спектр одночастичного гамильтониана (20) имеет вид

$$
\varepsilon_{+}=-D-2 I+H_{\|}, \quad \varepsilon_{0}=0, \quad \varepsilon_{-}=-D+2 I-H_{\|} .
$$

Из формул (21) видно, что при $D>2 I$ в продольном поле может произойти потеря устойчивости ФМ-фазы. Действительно, из равенства энергий основного 


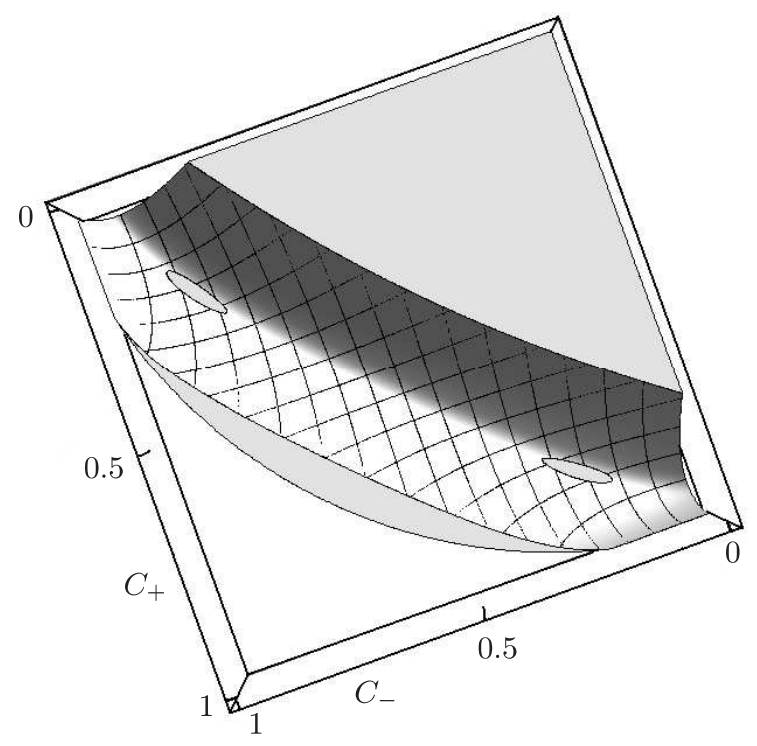

Рис. 5. Энергетический профиль $E_{\mathrm{gr}}\left(C_{+}, C_{-}\right)$в поперечном поле $H_{\perp}<H_{\mathrm{cr}}^{\perp}$. Положение минимумов показано эллипсами.

и возбужденного спиновых состояний, $\varepsilon_{+}=\varepsilon_{-}$, находим, что в поле $H_{\|}=2 I$ обменное и внешнее поля компенсируют друг друга. Это обеспечивает разрушение ФМ-состояния, поскольку в критической точке спины оказываются не связанными между собой. В итоге в продольном поле ориентационный фазовый переход в системе с $D>2 I$ путем когерентного вращения спинов исключен, и она ведет себя подобно изинговской. Другими словами, в продольном критическом поле имеет место фазовый переход I рода с критическим полем $H_{\mathrm{cr}}^{\|}=2 I$, который относится к магнитным КФП, индуцируемым внешним магнитным полем.

Эти особенности фазового перехода целиком вызваны действием ОА. Для одноосных ФМ с межионной анизотропией (или, что то же самое, анизотропным ОВ) любой величины происходит только ориентационный фазовый переход с вращением спинов (без какого-либо эффекта квантового сокращения).

\section{5. ПОПЕРЕЧНОЕ ПОЛЕ}

Рассмотрим намагничивание системы в поперечном магнитном поле, когда ненулевой будет только его составляющая $H_{\perp}$. Несмотря на то что для этого направления магнитного поля задача определения спиновых конфигураций может быть рассмотрена с использованием процедуры самосогласования, как в работах [8]-[15], [17]-[21], этот случай намагничивания также важно рассмотреть с точки зрения теории Ландау.

Действительно, из рис. 5 видно, что энергетический профиль $E_{\mathrm{gr}}\left(C_{+}, C_{-}\right)$в поперечном поле имеет два одинаковых минимума, расположенных симметрично в плоскости $C_{+}, C_{-}$(на рис. 5 положение минимумов показано эллипсами), что, в конеч- 
ном результате, приводит к непрерывному фазовому переходу, как в теории Ландау фазовых переходов II рода. В обоих минимумах оси квантования симметрично скошены к легкой оси. В однородном состоянии система будет находиться в одном из этих минимумов, и переходов из одного минимума в другой, если $H_{\|}=0$, быть не может. Поэтому в поперечном поле состояние ФМ-системы всегда будет ферромагнитным, устойчивым и равновесным. При возрастании величины напряженности магнитного поля минимумы сближаются, а ось квантования в собственной системе все больше отклоняется от легкой оси. В критическом поле $H_{\perp}=H_{\text {сr }}^{\perp}$ оба минимума сливаются, и седловая точка, разделяющая их, исчезает. При дальнейшем возрастании поля в системе будет только один минимум.

В поле $H_{\perp}=H_{\mathrm{cr}}^{\perp}$ проекция $\left\langle S_{z}\right\rangle$ становится равной нулю, что соответствует решению $\theta=\pi / 2$ уравнения (14), и отличной от нуля будет только проекция $\left\langle S_{x}\right\rangle$ вектора среднего спина, причем, как следует из уравнения $(13), \cos (2 \phi)<1$, где ось $X \uparrow \uparrow H_{\perp}$. Следовательно, при $H_{\perp}>H_{\text {cr }}^{\perp}$ система будет намагниченной вдоль поля. В поле $H_{\perp}<H_{\text {cr }}^{\perp}$ вектор среднего спина отклонен от легкой оси и не направлен вдоль поля.

Описание намагничивания одноосного ФМ с ОА в поперечном поле удобнее провести, используя собственную систему координат. Из уравнения (14) находим, что в состоянии с намагниченностью, скошенной к легкой оси, когда $0<\theta<\pi / 2$, величина этого угла определяется точным выражением

$$
\sin \theta=\frac{H_{\perp} \cos (2 \phi)}{D(1-\sin (2 \phi))} .
$$

Подставив его в уравнение (13), найдем, что средний спин в скошенном ФМ-состоянии зависит от поля согласно формуле

$$
\left\langle S_{\zeta}\right\rangle=\sqrt{1-\left(\frac{H_{\perp}^{2}}{4 I D}\right)^{2}} .
$$

Наконец, из формул (22) и (23) находим зависимость

$$
\sin \theta=\frac{H_{\perp}}{D} \frac{\sqrt{1+H_{\perp}^{2} /(4 I D)}}{\sqrt{1-H_{\perp}^{2} /(4 I D)}},
$$

справедливую при любых соотношениях между ОА и ОВ. Соответственно так же легко записываются проекции намагниченности на координатные оси:

$$
\begin{aligned}
& \left\langle S_{x}\right\rangle=\left\langle S_{\zeta}\right\rangle \sin \theta=\frac{H_{\perp}}{D}\left(1+\frac{H_{\perp}^{2}}{4 I D}\right) \\
& \left\langle S_{z}\right\rangle=\left\langle S_{\zeta}\right\rangle \cos \theta=\sqrt{1-\left(\frac{H_{\perp}^{2}}{4 I D}\right)^{2}-\frac{H_{\perp}^{2}}{D^{2}}\left(1+\frac{H_{\perp}^{2}}{4 I D}\right)^{2}} .
\end{aligned}
$$

Как и выше, обсудим намагничивание поперечным полем для двух случаев: $D / 2 I<1$ и $D / 2 I>1$, хотя заранее можно отметить, что качественных различий между результатами практически нет. 


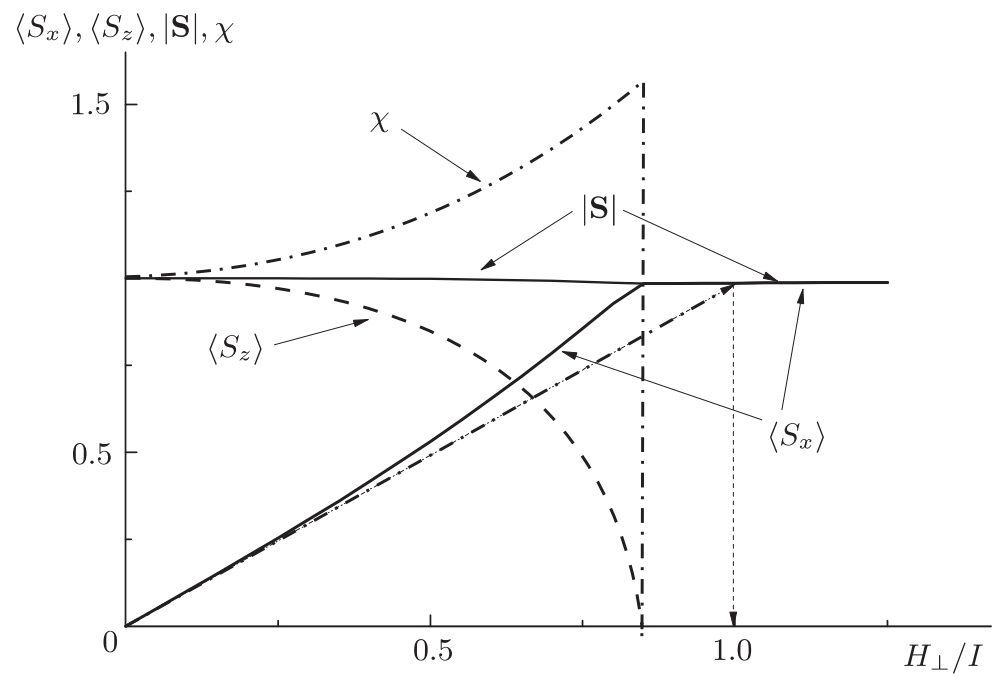

Рис. 6. Полевые зависимости для магнитной восприимчивости $\chi$, модуля среднего спина $|\mathbf{S}|$ и проекций среднего спина $\left\langle S_{x}\right\rangle,\left\langle S_{y}\right\rangle$ в поперечном поле $H_{\perp}$ при $D / I=1$.

При $D / 2 I \ll 1$ средний спин изменяется настолько слабо, что можно положить $\cos (2 \phi) \approx 1$. Тогда, как следует из формулы $(22)$, скос спина во всех полях линейно зависит от поля, а величина критического поля $H_{\mathrm{cr}}^{\perp} \approx D$.

При умеренных значениях ОА, когда $D / 2 I \leqslant 1$, модуль средней намагниченности при $H_{\perp} \rightarrow 0$ также достаточно слабо зависит от поля, и поэтому в малых полях $\left(H_{\perp} / D \ll 1\right)$ можно считать, что $\left\langle S_{z}^{2}\right\rangle \approx\left\langle S_{z}\right\rangle^{2}$. Восприимчивость этого начального участка (показана асимптотой на рис. 6), определенная в виде производной $\chi=d\left\langle S_{x}\right\rangle / d H_{\perp}$, дает гипотетическое критическое поле, равное $H_{\mathrm{cr}}^{\perp}=D$. В действительности, из хода полевых зависимостей $\left\langle S_{x}\right\rangle$ и $\left\langle S_{z}\right\rangle$, приведенных на рис. 6 , видно, что процесс скоса завершается раньше, поэтому $H_{\mathrm{cr}}^{\perp}<D$.

В случае $D / 2 I>1$ намагничивание в полях $H_{\perp} / D \ll 1$ также имеет квазиклассический характер, а магнитная восприимчивость $\chi=\left(d\left\langle S_{x}\right\rangle / d H_{\perp}\right)_{H_{\perp} \rightarrow 0}=1 / D$. Но, как видно из рис. 7 , при возрастании $H_{\perp}$, когда поле становится сопоставимым с $D$, в полевой зависимости среднего спина усиливается влияние квантовых эффектов, и его сокращение становится заметным. Кроме этого, в полях $H_{\perp}>H_{\mathrm{cr}}^{\perp}$ даже после того, как средний спин становится параллельным полю, модуль среднего спина не насыщен и имеется полевой ("парамагнитный") вклад в восприимчивость за счет полевого “вытягивания" среднего спина.

Найдем критическое поле, положив в формуле $(24)(\sin \theta)_{H_{x}=H_{\mathrm{cr}}^{\perp}}=1$. Нетрудно видеть, что

$$
H_{\mathrm{cr}}^{\perp}=\sqrt{\frac{D}{2}\left(\sqrt{D^{2}+24 I D+16 I^{2}}-4 I-D\right)} .
$$

Изменение величины $H_{\mathrm{cr}}^{\perp}(D)$ как функции константы ОА показано на рис. 8 . 


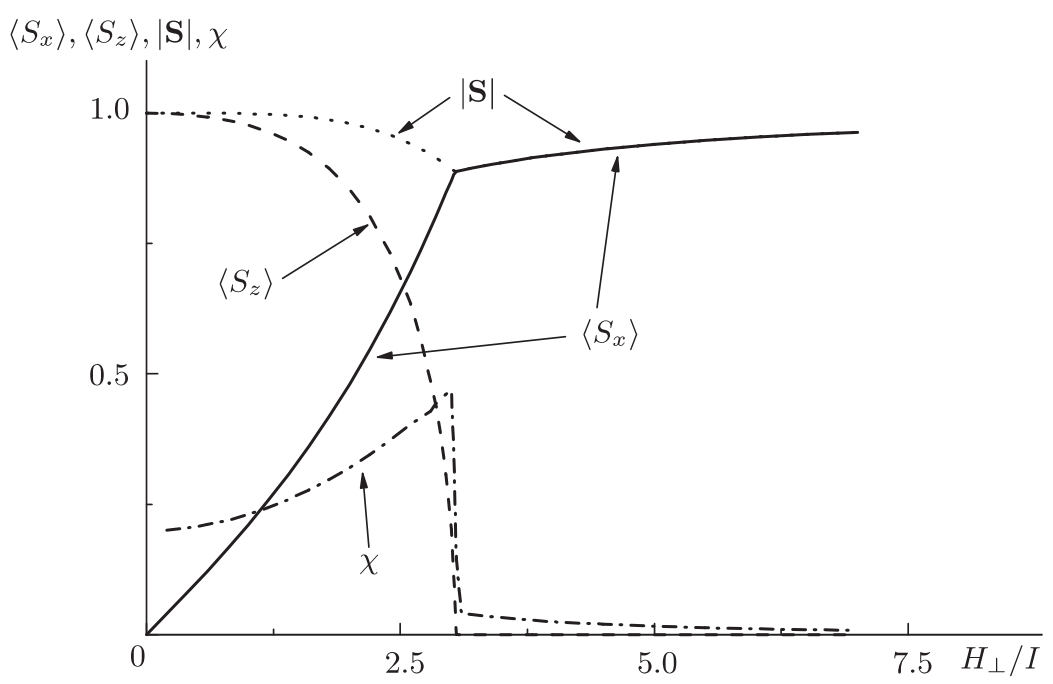

Рис. 7. Полевые зависимости для магнитной восприимчивости $\chi$, модуля среднего спина $|\mathbf{S}|$ и проекций среднего спина $\left\langle S_{x}\right\rangle,\left\langle S_{z}\right\rangle$ в поперечном поле $H_{\perp}$ при $D / I=5$.

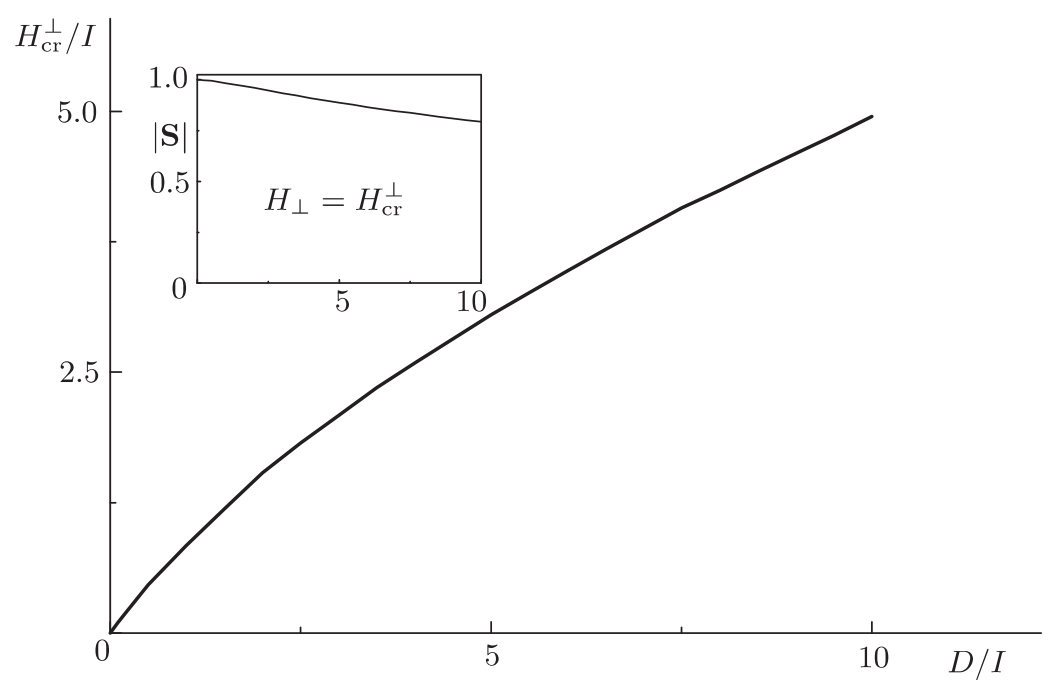

Рис. 8. Зависимость поперечного критического поля $H_{\mathrm{cr}}^{\perp}$ от константы ОА. На вставке показан ход зависимости модуля среднего спина $|\mathbf{S}|$ в критической точке при намагничивании в поперечном поле.

На вставке на рис. 8 показан ход зависимости модуля среднего спина от константы ОА в критической точке $H_{\perp}=H_{\mathrm{cr}}^{\perp}$. При $D / I \ll 1$ волновая функция $(3)$ в критической точке $H_{\perp}=H_{\text {сг }}^{\perp}$ стремится к виду $\psi=(1 / 2)|+1\rangle+(1 / \sqrt{2})|0\rangle+(1 / 2)|-1\rangle$, а потому средний спин в критической точке равен единице. При относительно больших $D$ 
$\left\langle S_{z}\right\rangle, C_{+}, C_{-}, C_{+}-C_{-}$

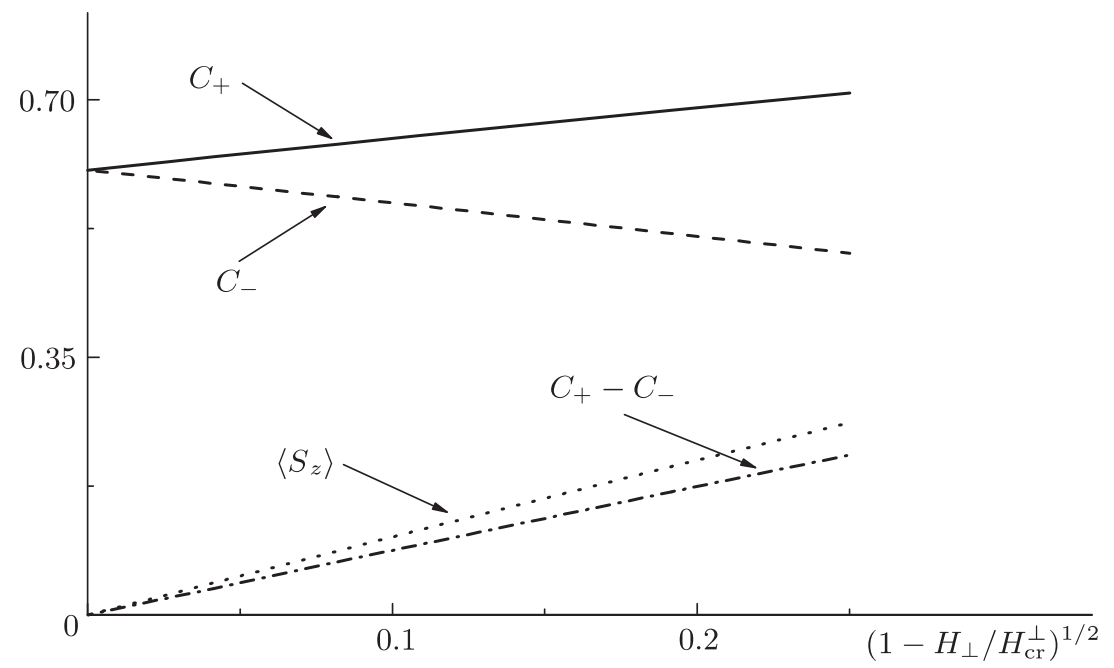

Рис. 9. Полевые зависимости для параметров волновой функции $C_{+}, C_{-}$, а также их разности $C_{+}-C_{-}$и проекции среднего спина $\left\langle S_{z}\right\rangle$ от $\sqrt{1-H_{\perp} / H_{\mathrm{cr}}^{\perp}}$ в области критического поля.

в поле $H_{\perp}=H_{\text {cr }}^{\perp}$ выполняется условие $C_{+}=C_{-}>1 / 2$, что приводит к сокращению поперечной проекции среднего спина и его модуля в критической точке.

На рис. 9 показано полевое поведение параметров $C_{+}$и $C_{-}$вблизи критического поля $H_{\mathrm{cr}}^{\perp}$. Из этого рисунка видно, что они линейно зависят от корня $\sqrt{1-H_{\perp} / H_{\mathrm{cr}}^{\perp}}$. В окрестности критического поля при $H_{\perp} \rightarrow H_{\mathrm{cr}}^{\perp}$ можно записать

$$
\left\langle S_{z}\right\rangle \approx 2 C_{+}\left(H_{\perp}=H_{\mathrm{cr}}^{\perp}\right)\left(C_{+}-C_{-}\right) .
$$

Тогда из рис. 9 видно, что в критической области полевая зависимость разности коэффициентов $C_{+}$и $C_{-}$также пропорциональна корню $\sqrt{1-H_{\perp} / H_{\mathrm{cr}}^{\perp}}$ и, таким образом (см. формулу $(25))$, величина $\left\langle S_{z}\right\rangle \sim \sqrt{1-H_{\perp} / H_{\mathrm{cr}}^{\perp}}$ имеет такое же критическое поведение, как и параметры волновой функции.

В итоге для всех значений параметра ОА КФП II рода в поперечном поле является спин-ориентационным фазовым переходом, проявляющимся в критическом характере отклонения вектора средней намагниченности от трудного направления. Как видно, этот фазовый переход также может быть описан минимизацией по параметрам волновой функции, однако в этом случае критическое поведение параметров $C_{+}$, $C_{-}$связано с изменением направления средней намагниченности.

\section{6. УГЛОВАЯ ЗАВИСИМОСТЬ КРИТИЧЕСКОГО ПОЛЯ}

Намагничивание $\Phi \mathrm{M} \mathrm{c} \mathrm{OА} \mathrm{в} \mathrm{поперечном} \mathrm{поле} \mathrm{является} \mathrm{особым} \mathrm{случаем,} \mathrm{потому}$ что только для такого направления поля скос намагниченности непрерывен и скачков намагниченности при перемагничивании нет. В поле, направленном под произвольным углом к легкой оси (обозначим этот угол $\beta$ ), минимум, отвечающий устой- 


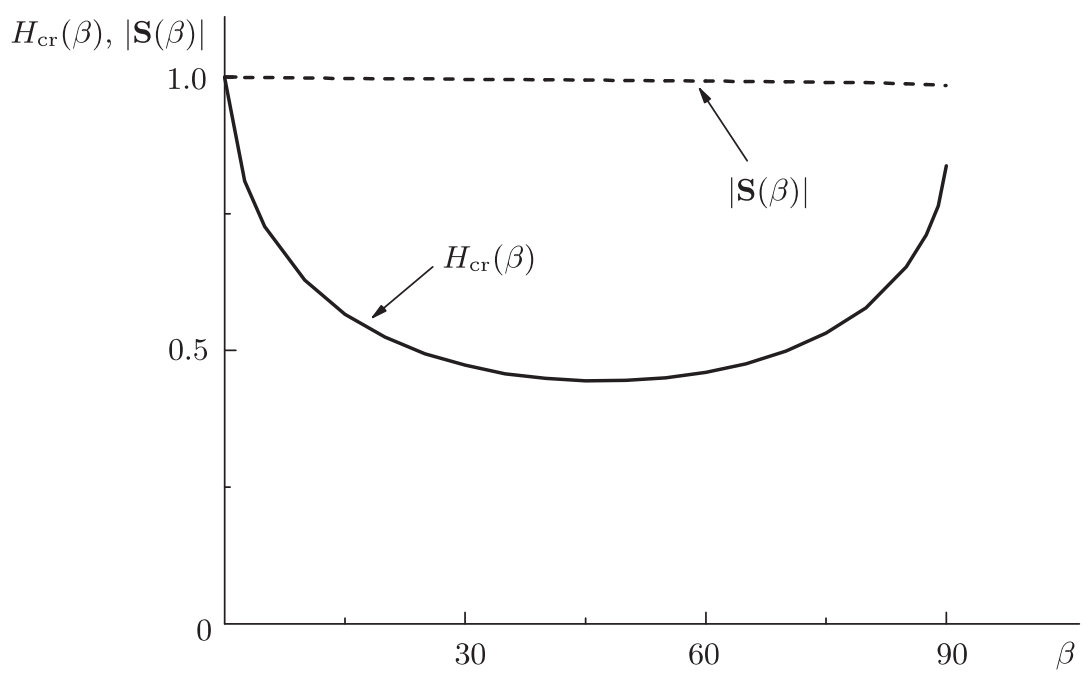

Рис. 10. Графики зависимостей критического поля $H_{\mathrm{cr}}(\beta)$ и модуля среднего спина $|\mathbf{S}(\beta)|$ от угла $\beta$ между вектором магнитного поля и легкой осью $Z$ при $D / I=1$.

чивому ФМ-состоянию, и минимум, отвечающий неустойчивому ФМ-состоянию, имеют разные значения энергии. Поэтому для каждого направления внешнего магнитного поля имеется свое критическое поле $H_{\mathrm{cr}}(\beta)$, в котором скачкообразно происходит переход от неустойчивого ФМ-состояния к устойчивому.

Как уже говорилось, при $D / I \ll 1$ выполняется квазиклассическое приближение, и угловая зависимость для $H_{\mathrm{cr}}(\beta)$ будет описываться астроидой Стонера-Вольфарта [39], [40]. При сопоставимых величинах констант $D$ и $I$ угловая зависимость для $H_{\mathrm{cr}}(\beta)$ будет отличаться от этой астроиды, причем характер изменения хода кривой $H_{\mathrm{cr}}(\beta)$ зависит от величин ОА и обмена.

Для примера на рис. 10 показана зависимость $H_{\mathrm{cr}}(\beta)$, полученная для случая $D / I=1$. Видно, что отношение $H_{\mathrm{cr}}(\beta=\pi / 4) / H_{\mathrm{cr}}^{\|} \approx 0.44$. Для астроиды отношение величин этих полей равно 0.5. Видно также, что ход зависимости $H_{\mathrm{cr}}(\beta)$ несимметричен относительно точки $\beta=\pi / 4$, причем $H_{\mathrm{cr}}(\beta \rightarrow \pi / 2)<H_{\mathrm{cr}}(\beta \rightarrow 0)$. При $D / I=1$ модуль среднего спина неравновесного ФМ-состояния, определенный в критической точке, слабо зависит от угла $\beta$. Максимальное "сокращение" длины вектора среднего спина происходит при $\beta \rightarrow \pi / 2$, и для $D / I=1$ оно составляет лишь $1.5 \%$.

На рис. 11 показана угловая зависимость $H_{\mathrm{cr}}(\beta)$, для случая $D / I=5$. Теперь отношение $H_{\mathrm{cr}}(\beta=\pi / 4) / H_{\mathrm{cr}}^{\|} \approx 0.75$. Кроме того, изменяется характер несимметричности зависимости $H_{\mathrm{cr}}(\beta)$ и имеет место неравенство $H_{\mathrm{cr}}(\beta \rightarrow \pi / 2)>H_{\mathrm{cr}}(\beta \rightarrow 0)$. Как и в предыдущем случае, наибольшее "сокращение" длины вектора среднего спина происходит при $\beta \rightarrow \pi / 2$; например, при $D / I=5$ оно составляет $11 \%$.

Таким образом, квантовые эффекты влияют на угловую зависимость критического поля перехода от неустойчивого к устойчивому ФМ-состоянию, причем, как оказалось, в случае ОА, значительно превосходящей ОВ, величина критического 


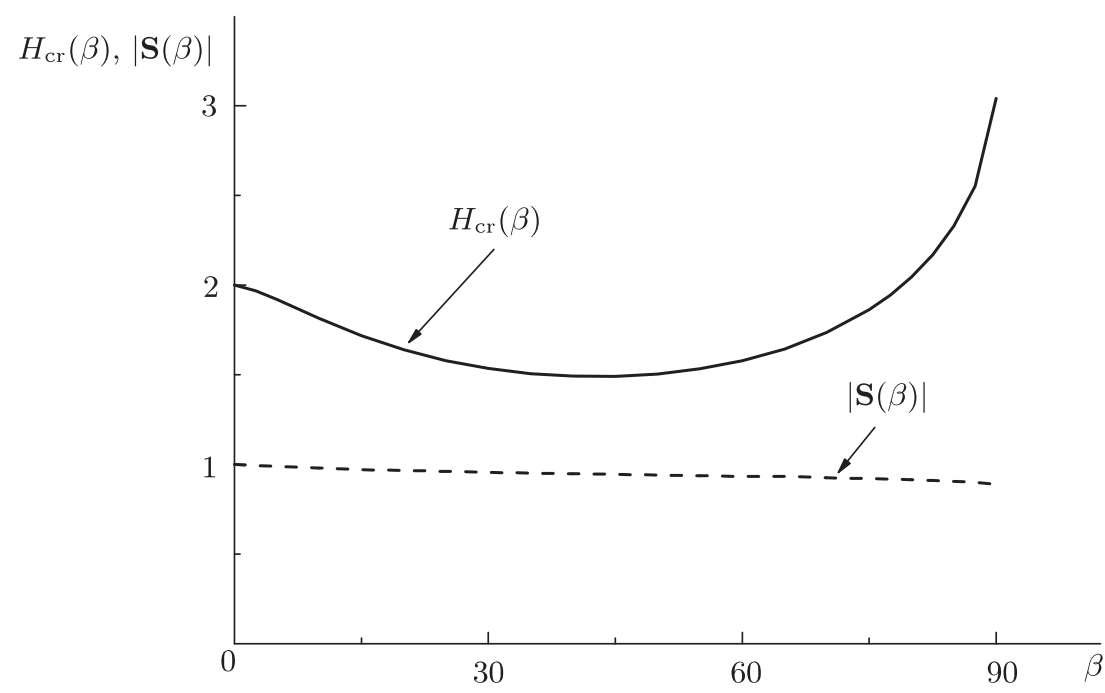

Рис. 11. Графики зависимостей критического поля $H_{\mathrm{cr}}(\beta)$ и модуля среднего спина $|\mathbf{S}(\beta)|$ от угла $\beta$ между вектором магнитного поля и легкой осью $Z$ при $D / I=5$.

поля испытывает аномальный рост для углов, близких к направлению трудного намагничивания.

Заметим, наконец, что случай, когда ОА значительно больше ОВ, может иметь место для так называемого суперферромагнитного состояния гранулярных магнитных пленок [42], [43]. В этом состоянии магнитных однодоменных наночастиц с одноосной анизотропией может наблюдаться аномалия угловой зависимости критического поля для углов, близких к направлению трудного намагничивания. Эта аномалия вызвана тем, что при рассмотрении ансамбля частиц их анизотропию формально можно рассматривать как одноионную. Однако в отличие от рассмотренной выше квантовой системы в гранулярных магнитных системах "ОА" наногранул и их "ОВ" записываются в виде функционалов, зависящих от классического вектора магнитного момента частицы, а полученная в работах [42], [43] аномальная угловая зависимость для критического поля наблюдается при конечных температурах. При этом, как оказалось, описанный нами квантовый эффект возрастания критического поля для трудного направления и квазиклассический температурный (учитывающий тепловые флуктуации для направления магнитного момента) эффект возрастания такого критического поля качественно подобны и реализуются в случае сильной ОА, когда она в несколько раз превышает ОВ.

\section{7. ЗАКЛЮЧЕНИЕ}

На основе квантово-механического подхода показано, что даже в одноосном ФМ с ОА можно ожидать проявления квантовых эффектов в статических измерениях, в частности при изучении процессов намагничивания в продольном магнитном поле. 
При этом расчет квантовых поправок проведен путем минимизации энергии основного состояния по параметрам волновой функции, что отвечает духу теории Ландау фазовых переходов.

Оказалось, что характер перемагничивания одноосного $Ф \mathrm{M} \mathrm{c} S=1$ в продольном поле существенно зависит от величины константы ОА. Если она меньше ОВ, то переход из неустойчивого ФМ-состояния в устойчивое происходит путем вращения вектора намагниченности. Это означает, что в критической точке все спины однородно разворачиваются к направлению с выгодной ориентацией средней намагниченности. Но даже при таком вращении среднего спина, когда он отклоняется от легкой оси, может иметь место наблюдаемое квантовое сокращение его модуля. Если же ОА больше ОВ, то продольное критическое поле равно величине обменного поля (равного полю устойчивости ФМ-фазы). Для сильно анизотропного ФМ при перемагничивании в критическом поле однородного разворота спинов не происходит, чему препятствует более сильная (не превышающая ОВ) ОА. Соответствующий фазовый переход является примером магнитного КФП I рода.

При ориентационном перемагничивании поперечным магнитным полем таких ограничений на величины ОА и ОВ не возникает. Для всех значений ОА в поперечном магнитном поле происходит непрерывный разворот намагниченности, на который "налагается" квантовое сокращение, особенно заметное при больших значениях ОА. Квантовое сокращение среднего спина оказывается наиболее существенным в критической точке.

Показано также, что благодаря последовательному учету ОА продольное критическое поле не равно поперечному критическому полю. Для случая $D / 2 I<1$ первое больше второго; если же ОА гораздо больше ОВ, то реализуется обратное неравенство для величин критических полей. В настоящей работе также продемонстрировано, что в легкоосном $Ф \mathrm{M} \mathrm{с} \mathrm{большой} \mathrm{ОА} \mathrm{угловая} \mathrm{зависимость} \mathrm{критического} \mathrm{поля} \mathrm{не}$ следует астроиде Стонера-Вольфарта. Нелинейность, порождаемая ОА, приводит к значительной асимметрии в угловой зависимости критического поля, разделяющего неустойчивое и устойчивое ФМ-состояния. При ОА, превосходящей ОВ, имеет место аномалия угловой зависимости критического поля, величина которого возрастает в направлении трудного намагничивания. Можно надеяться, что отмеченные квантовые эффекты могут проявиться при исследовании такого известного и относительно простого для экспериментального изучения процесса, как намагничивание (или перемагничивание) анизотропных магнетиков.

Благодарности. Работа выполнена при частичной поддержке Целевой программы фундаментальных исследований Отделения физики и астрономии НАН Украины.

\section{Список литературы}

[1] Е. М. Лифшиц, ЖЭТФ, 15:3 (1945), 97-107.

[2] С. В. Вонсовский, УФН, 37:1 (1949), 1-64.

[3] З. А. Казей, Н. П. Колмакова, Р. З. Левитин, В. В. Платонов, А. А. Сидоренко, О. М. Таценко, Писъма в ЖЖЭТФ, 65:9 (1997), 691.

[4] О. Б. Заславский, В. В. Ульянов, Ю. В. Василевская, ФНT, 24:7 (1998), 627-634. 
[5] М. С. Тагиров, Д. А. Таюрский, ФНT, 28:3 (2002), 211-234.

[6] З. А. Казей, В. В. Снегирев, Ж.-М. Брото, Х. Ракото, Писъма в ЖКЭТФ, 87:12 (2008), $787-791$.

[7] М. И. Кобец, Е. Н. Хацько, К. Г. Дергачев, П. С. Калинин, ФНТ, 36:7 (2010), 767-775.

[8] В. М. Локтев, В. С. Островский, УФЖ, 23:10 (1978), 1708-1717.

[9] Ф. П. Онуфриева, ЖЭТФ, 80:6 (1981), 2372-2379.

[10] В. Г. Борисенко, Ю. В. Переверзев, ФНТ, 11 (1985), 730-735.

[11] В. В. Вальков, Т. А. Валькова, С. Г. Овчинников, ЖЖЭТФ, 88 (1985), 550-561.

[12] В. С. Островский, ЖЭТФ, 91 (1986), 1690-1701.

[13] Ю.Н. Мицай, Ю. А. Фридман, ТМФ, 81:2 (1989), 263-270.

[14] В. М. Локтев, В. С. Островский, ФНТ, 20:10 (1994), 983-1016.

[15] V. Yu. Irkhin, Yu. P. Irkhin, Phys. Stat. Sol., 193 (1994), 9.

[16] В. М. Калита, В. М. Локтев, ФНТ, 28:12 (2002), 1244-1250; ФTT, 45:8 (2003), 1450-1455; $47: 4$ (2005), 666-672.

[17] Yu. A. Fridman, O. A. Kosmachev, Ph. N. Klevets, JMMM, 320:3-4 (2008), 435-449.

[18] Ю. А. Фридман, Д. А. Матюнин, ФТT, 50:4 (2008), 669-674.

[19] Yu. A. Fridman, D. A. Matunin, Ph. N. Klevets, O. A. Kosmachev, JMMM, 321:22 (2009), 3782-3794.

[20] В. В. Вальков, С. Г. Овчинников, Квазичастицы в сильно коррелированных системах, Изд-во СО РАН, Новосибирск, 2001.

[21] V.Yu. Irkhin, Yu. P. Irkhin, Electronic Structure, Correlation Effects and Properties of dand $f$-Metals and Their Compounds, Cambridge Internat. Sci. Publ., Cambridge, 2007.

[22] Р. С. Гехт, УФH, 159:2 (1989), 261-296.

[23] M.F. Collins, O.A. Petrenko, Can. J. Phys., 75:9 (1997), 605-655, arXiv: cond-mat/9706153.

[24] Б. С. Думеш, УФН, 170:4 (2000), 403-418.

[25] V.S. Zapf, D. Zocco, B. R. Hansen, M. Jaime, N. Harrison, C. D. Batista, M. Kenzelmann, C. Niedermayer, A. Lacerda, A. Paduan-Filho, Phys. Rev. Lett., 96:7 (2006), 077204, 4 pp., arXiv: cond-mat/0505562.

[26] S. A. Zvyagin, J. Wosnitza, C.D. Batista, M. Tsukamoto, N. Kawashima, J. Krzystek, V.S. Zapf, M. Jaime, N. F. Oliveira, Jr., A. Paduan-Filho, Phys. Rev. Lett., 98:4 (2007), 047205, 4 pp., arXiv: cond-mat/0701750.

[27] V. S. Zapf, V. F. Correa, P. Sengupta, C. D. Batista, M. Tsukamoto, N. Kawashima, P. Egan, C. Pantea, A. Migliori, J. B. Betts, M. Jaime, A. Paduan-Filho, Phys. Rev. B, 77:2 (2008), 020404(R), 4 pp.

[28] O. Chiatti, A. Sytcheva, J. Wosnitza, S. Zherlitsyn, A. A. Zvyagin, V.S. Zapf, M. Jaime, A. Paduan-Filho, Phys. Rev. B, 78:9 (2008), 094406, 5 pp., arXiv: 0809.1515).

[29] В.М.Калита, В.М.Локтев, ЖЭТФ, 125:5 (2004), 1149-1158; ФНТ, 31:7 (2005), 815; Писъма в ЖЖЭТФ, 91:4 (2010), 196-200.

[30] В. М. Калита, В. М. Локтев, Писъма в ЖКЭТФ, 93:9 (2011), 592-596.

[31] V. M. Kalita, I. M. Ivanova, V. M. Loktev, Phys. Rev. B, 78:10 (2008), 104415, 10 pp., arXiv: 0707.4227.

[32] Л. Неель, Известия АН СССР. Сер. физ., 21:6 (1957), 890-903.

[33] К. П. Белов, А. К. Звездин, А. М. Кадомцева, Р. З. Левитин, Ориентационные переходъ в редкоземелъных магнетиках, Наука, М., 1979.

[34] В. Г. Барьяхтар, И. М. Витебский, Д. А. Яблонский, ФТТ, 19 (1977), 2135.

[35] С. В. Тябликов, Методы квантовой теории магнетизма, Наука, М., 1975.

[36] В. Ф. Гантмахер, В. Т. Долгополов, УФН, 178:1 (2008), 3-24.

[37] Б. А. Иванов, Писъма в ЖКЭТФ, 84:2 (2006), 90-94. 
[38] В. И. Бутрим, Б. А. Иванов, А. С. Кузнецов, Писъма в ЖЖЭТФ, 92:3 (2010), 172-176.

[39] E. C. Stoner, E. P. Wohlfarth, Phil. Trans. R. Soc. Lond. A, 240:826 (1948), 599-642.

[40] Л. Д. Ландау, Е. М. Лифшиц, Теоретическая физика, т. 8: Электродинамика сплошных сред, Наука, М., 1982.

[41] М. Ю. Ковалевский, ФНТ, 36:8-9 (2010), 1006-1012.

[42] A. A. Timopheev, V.M. Kalita, S. M. Ryabchenko, A. F. Lozenko, P. A. Trotsenko, A. V. Los, M. Munakata, J. Appl. Phys., 108:5 (2010), 053902, 8 pp.

[43] С. М. Рябченко, А.А. Тимофеев, В.М. Калита, А.Ф. Лозенко, П.А. Троценко, В. А. Стефанович, М. Мунаката, ФНТ, 36:8 (2010), 861-875.

Поступила в редакцию 25.01.2012, после доработки 8.02.2012 